\title{
Efficacy of Nebulized Glycopyrrolate on Lung Hyperinflation in Patients with COPD
}

Thomas M. Siler · Claire Hohenwarter · Kuangnan Xiong ·

Kenneth Sciarappa $\cdot$ Shahin Sanjar · Sanjay Sharma

Received: May 7, 2021 / Accepted: June 21, 2021 / Published online: July 7, 2021

(C) The Author(s) 2021

\section{ABSTRACT}

Introduction: Lung hyperinflation in chronic obstructive pulmonary disease (COPD) is associated with activity limitation, impaired cardiac output, and mortality. Several studies have demonstrated that long-acting muscarinic antagonists (LAMAs) delivered by dry powder inhalers can promote lung deflation; however, the potential of nebulized LAMAs on improving hyperinflation in COPD is currently unknown. Methods: This single-center, randomized, double-blind, two-way crossover study (NCT04155047) evaluated the efficacy of a single dose of nebulized LAMA [glycopyrrolate (GLY) $25 \mu \mathrm{g}$ ] versus placebo in patients with COPD and lung hyperinflation. Patients with moderate-to-severe COPD and a residual volume $(\mathrm{RV}) \geq 130 \%$ of predicted normal were included. The primary endpoint was changed from baseline in $\mathrm{RV}$ at $6 \mathrm{~h}$ post-treatment. Other endpoints included changes from

Supplementary Information The online version contains supplementary material available at https:// doi.org/10.1007/s41030-021-00166-5.

T. M. Siler

Midwest Chest Consultants, St Charles, MO, USA

C. Hohenwarter · K. Xiong · K. Sciarappa - S. Sanjar

. S. Sharma ( $\square)$

Sunovion Pharmaceuticals Inc., Marlborough, MA, USA

e-mail: sanjay.sharma@sunovion.com baseline in spirometric and plethysmographic measures up to $6 \mathrm{~h}$ post-treatment.

Results: A total of 22 patients (mean pre-bronchodilator RV, $153.7 \%$ of predicted normal) were included. The primary objective of the study was not met; the placebo-adjusted least squares (LS) mean [95\% confidence interval (CI) change from baseline in RV with GLY at $6 \mathrm{~h}$ post-treatment was -0.3231 ( -0.711 to 0.066 ); $p=0.0987]$. A post hoc evaluation of the primary analysis was conducted after excluding a single statistical outlier; substantial improvements in RV with GLY compared with placebo was observed after exclusion of this outlier [placebo-adjusted LS mean change from baseline $(95 \% \mathrm{CI})$ in $\mathrm{RV}$ was $-0.446 \mathrm{l}(-0.741$ to - 0.150)]. Improvements from baseline were also observed with GLY compared with placebo in spirometric and plethysmographic measures up to $6 \mathrm{~h}$ post-treatment. GLY was generally safe, and no new safety signals were detected.

Conclusions: This is the first study to evaluate the effect of nebulized GLY on lung deflation. Nebulized GLY resulted in marked improvements in RV up to $6 \mathrm{~h}$ post-treatment, compared with placebo. Improvements were also observed with GLY in spirometric and plethysmographic parameters of lung function.

Trial Registration: ClinicalTrials.gov identifier, NCT04155047. 
Keywords: Body plethysmography; COPD; Hyperinflation; Lung deflation; Nebulized glycopyrrolate; Residual volume

Infographic:

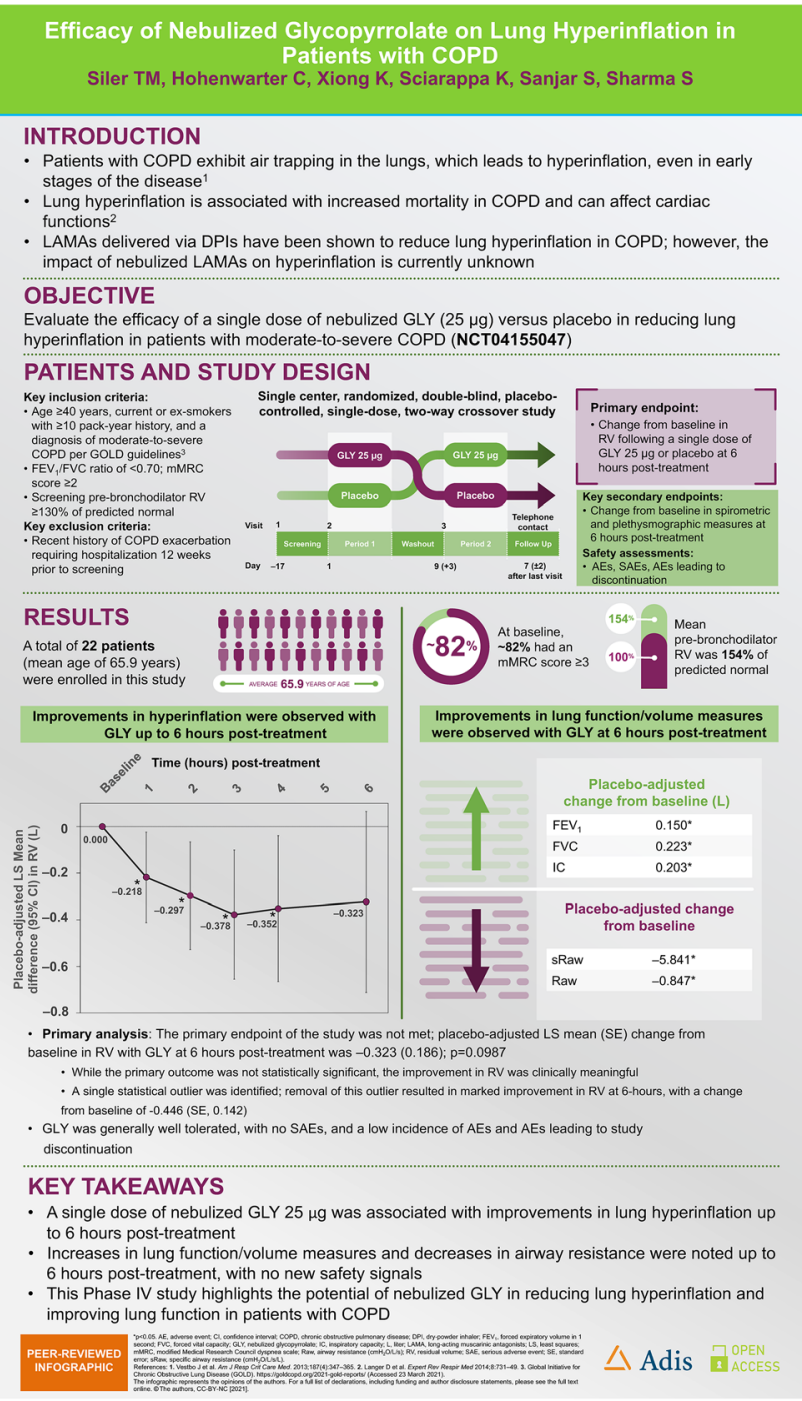

\section{Key Summary Points}

\section{Why carry out this study?}

Patients with chronic obstructive pulmonary disease (COPD) suffer from dyspnea (or shortness of breath); dyspnea is often due to an increase in the amount of gas in the lungs at the end of expiration (known as hyperinflation).

This phase IV study evaluated the efficacy of a single dose of nebulized glycopyrrolate (GLY $25 \mu \mathrm{g}$; a bronchodilator) versus placebo in reducing lung hyperinflation in patients with moderate-to-severe COPD.

\section{What was learned from the study?}

Nebulized GLY showed substantial improvements in hyperinflation as measured by residual volume after $6 \mathrm{~h}$ of treatment, compared with placebo. This was accompanied by increases in forced expiratory volume in $1 \mathrm{~s}\left(\mathrm{FEV}_{1}\right)$ and inspiratory capacity, and reduced airway resistance (Raw and sRaw).

This study highlights the potential of nebulized GLY in reducing lung hyperinflation and improving lung function in patients with COPD.

\section{DIGITAL FEATURES}

This article is published with digital features, including an infographic, to facilitate understanding of the article. To view digital features for this article go to https://doi.org/10.6084/ m9.figshare.14815074.

\section{INTRODUCTION}

Chronic obstructive pulmonary disease (COPD) is characterized by persistent respiratory symptoms and airflow limitation [1]. COPD is a result 
of airway and/or alveolar abnormalities, which typically occur from exposure to noxious particles or gases, although genetic factors may also play a role [1]. The most common respiratory symptoms in COPD include dyspnea, cough, and/or sputum production [1]. Patients with COPD also exhibit air trapping in the lungs, which leads to an increase in residual volume $(\mathrm{RV})$, even in early stages of the disease [2]; while there is no precise definition for air trapping, $\mathrm{RV} \geq 20 \%$ of the predicted normal levels is considered significant [3]. Further, as COPD worsens, airflow limitation increases, and lung hyperinflation occurs [2]. RV and functional residual capacity (FRC) progressively increase as COPD worsens [3]. Lung hyperinflation is associated with increased mortality in COPD, and can affect cardiovascular functions [4-7].

Long-acting $\beta_{2}$-agonists (LABAs) and longacting muscarinic antagonists (LAMAs) are the mainstay for bronchodilator therapy. LAMAs are considered to be the cornerstones of monotherapy [1], and several studies have explored the effects of a LAMA (e.g., tiotropium, aclidinium bromide) delivered via dry-powder inhalers (DPIs) twice-daily (BID) on lung deflation $[8,9]$. The ACTIVATE study assessed the effect of aclidinium bromide/formoterol $12 \mu \mathrm{g}$ BID delivered via a DPI in 267 patients with moderate-to-severe COPD; following 4 weeks of treatment, improvements in lung hyperinflation and exercise tolerance were observed with aclidinium bromide/formoterol compared with placebo [9]. In addition, LAMA/DPIs have also been shown to improve hyperinflation in COPD when administered once-daily (QD) [10-16]. While LAMAs delivered via DPIs may improve hyperinflation in COPD, the impact of nebulized LAMAs on hyperinflation is currently unknown. Pulmonary drug delivery via nebulization has documented advantages over DPIs, as it allows for greater delivery of the drug to the lungs, and may be suitable for patients with COPD who have difficulty using DPIs because of coordination difficulties and low inspiratory flow rates (e.g., elderly patients) [17].

Glycopyrrolate inhalation solution (GLY; Lonhala ${ }^{\circledR}$, Sunovion Pharmaceuticals Inc., Marlborough, MA, USA) $25 \mu \mathrm{g}$ BID delivered by

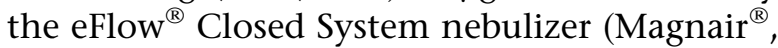

PARI Pharma GmbH, Starnberg, Germany) [18] was the first nebulized LAMA to be approved by the US Food and Drug Administration for the long-term maintenance treatment of airflow obstruction in patients with moderate-to-verysevere COPD in December 2017 [19]. This approval was based on the efficacy and safety demonstrated by the 12 -week, replicate, placebo-controlled Glycopyrrolate for Obstructive Lung Disease Via Electronic Nebulizer (GOLDEN) 3 and GOLDEN 4 (NCT02347761 and NCT02347774, respectively), and the 48-week GOLDEN 5 (NCT02276222) phase III randomized clinical trials $[20,21]$. Magnair ${ }^{\circledR}$ generates GLY aerosols in the respirable range $(1-5 \mu \mathrm{m})$ in vitro, allowing efficient pulmonary deposition [18, 22-24]. The objective of the current study was to characterize the effects of a single dose of nebulized LAMA (GLY $25 \mu \mathrm{g}$ ) on hyperinflation in patients with moderate-tosevere COPD.

\section{METHODS}

\section{Study Design}

This was a single-center (United States), randomized, double-blind, placebo-controlled, single-dose, two-way crossover, phase IV study (NCT04155047) conducted between November 7, 2019, and March 31, 2020. The study consisted of a screening period, a randomized twoway cross-over treatment period, and a followup $7( \pm 2)$ days after the last dose (Fig. 1). Patients were randomized to the crossover treatment sequences ( $\mathrm{B} B$ or $\mathrm{BA}$ ) in a 1:1 ratio, to receive two single doses of GLY $25 \mu \mathrm{g}$ and placebo, separated by a 7-day washout period. In sequence $A B$, patients received GLY $25 \mu \mathrm{g}$ in treatment period 1 and placebo in treatment period 2; in sequence $\mathrm{BA}$, patients received placebo in treatment period 1, followed by GLY $25 \mu \mathrm{g}$ in treatment period 2.

During treatment period 1 (day 1), a single dose of GLY $25 \mu \mathrm{g} /$ placebo was prepared by the clinical staff and self-administered by the patients in the morning according to the randomization schedule. To maintain blinding, clinical staff who were unblinded to the 

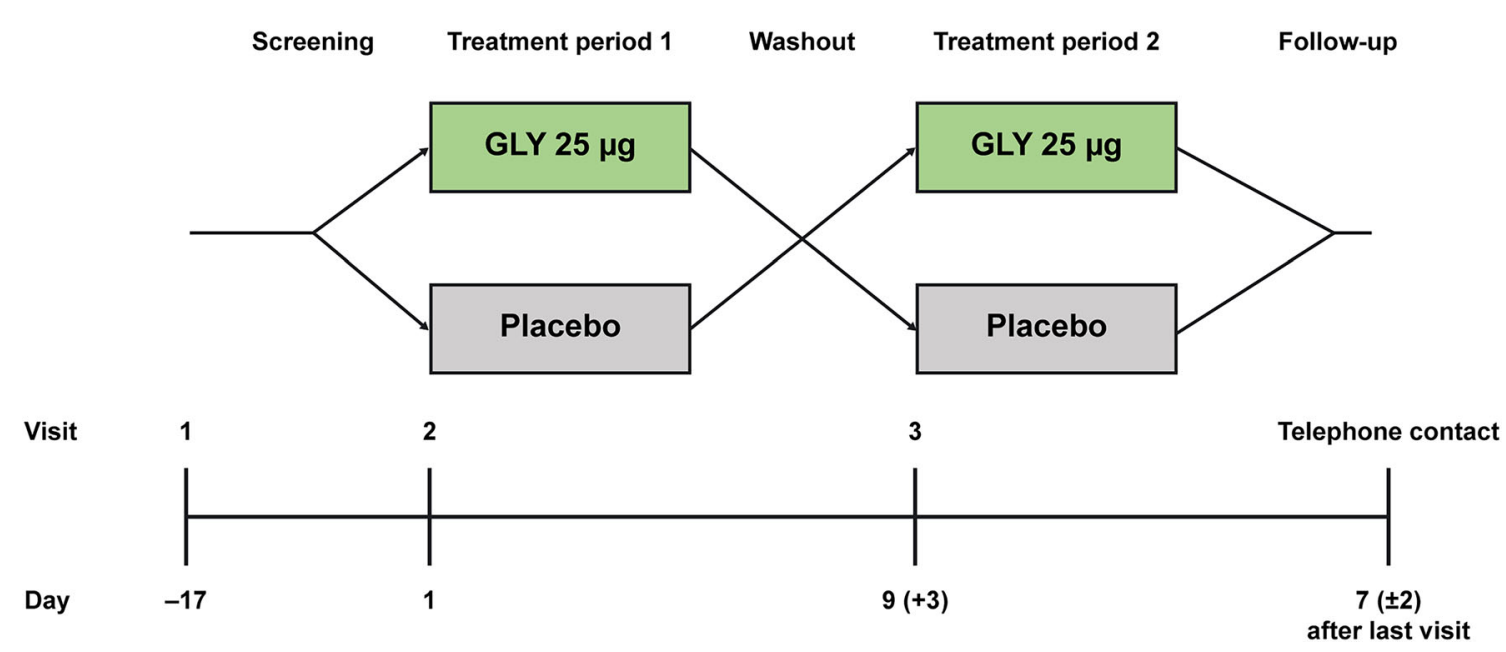

Fig. 1 SUN101-402 double-blind, two-way crossover study design. GLY glycopyrrolate

treatment performed the randomization and assisted with study drug administration but did not perform any other study procedures. Each patient was assigned a nebulizer, which was operated and sanitized according to the manufacturer's instructions by the site clinical staff. Patients returned to the clinical site on day 9 $(+3)$ for treatment period 2, and a single dose of GLY $25 \mu \mathrm{g} /$ placebo was prepared by the clinical staff and self-administered by the patients in the morning (within $1 \mathrm{~h}$ of treatment period 1 dosing time) according to the assigned treatment sequence.

Spirometry measurements were conducted at screening during both treatment periods (day 1 and day 9; 45 min pre-treatment and $6 \mathrm{~h}$ posttreatment) in accordance with the American Thoracic Society/European Respiratory Society (ATS/ERS) 2005 guidelines [25]. Plethysmography measurements were performed at screening during both treatment periods (day 1 and day 9; $45 \mathrm{~min}$ pre-treatment and $1,2,3,4$, and $6 \mathrm{~h}$ post-treatment), and were conducted in accordance with the current ATS/ERS 2005 guidelines) [26]. Plethysmography was performed prior to spirometry when time-points coincided.

The study (SUN101-402: project approval number 39173) protocol was approved by Quorum Review institutional review board prior to enrollment, and was conducted in accordance with the protocols of the International Conference on Harmonization Good Clinical
Practice guidelines, and Declaration of Helsinki. All patients provided written informed consent before participating in this study.

\section{Participants}

Male or female patients, $\geq 40$ years, current or ex-smokers with a $\geq 10$ pack-year history, and a clinical diagnosis of COPD were eligible to participate in the study. Additional inclusion criteria at screening were a pre-bronchodilator RV of $\geq 130 \%$ of predicted normal, moderate-tosevere COPD according to GOLD guidelines [post-bronchodilator (following inhalation of ipratropium bromide $68 \mu \mathrm{g}$ ) forced expiratory volume in $1 \mathrm{~s} \quad\left(\mathrm{FEV}_{1}\right)$ between $\geq 30 \%$ and $<80 \%$ of predicted normal] [1], $\mathrm{FEV}_{1} /$ forced vital capacity (FVC) ratio of $<0.70$, and a modified Medical Research Council Dyspnea Scale (mMRC) score of $\geq 2$. Key exclusion criteria included a recent history of COPD exacerbation requiring hospitalization 12 weeks prior to screening and inability to have plethysmography lung volume testing. Rescue medication (albuterol) was provided by the site during the study.

\section{Endpoints}

The primary endpoint was change from baseline in $\mathrm{RV}$ following a single dose of GLY $25 \mu \mathrm{g}$ or 
placebo at 6-h post-treatment. Other endpoints included change from baseline in spirometric $\left(\mathrm{FEV}_{1}\right.$ and $\left.\mathrm{FVC}\right)$, and plethysmography measures up to $6 \mathrm{~h}$ post-treatment. Plethysmography measurements included inspiratory capacity (IC), expiratory reserve volume (ERV), functional residual capacity (FRC), total lung capacity (TLC), airway resistance (Raw; $\mathrm{cmH}_{2} \mathrm{O} /$ $\mathrm{l} / \mathrm{s}$ ), and specific airway resistance (sRaw; $\left.\mathrm{cmH}_{2} \mathrm{O} / \mathrm{l} / \mathrm{s} / \mathrm{l}\right) ;$ additional measures included standardized area under the curve (AUC) at 3, 4, and $6 \mathrm{~h}$ post-treatment for the aforementioned parameters. Pearson's correlations between plethysmography endpoints with $\mathrm{FEV}_{1}$ and FVC by treatment group were estimated at 6 -h posttreatment. Safety assessments included the monitoring of adverse events (AEs), serious adverse events (SAEs), vital signs, and electrocardiogram (ECG). AEs were coded using MedDRA v22.1, and summarized by treatment, system organ class, and preferred term.

Exploratory endpoints included evaluation of the feasibility of using a biosensor, VitalPatch $^{\circledR}$, in combination with the accelerateIQ ${ }^{\circledR}$ platform. The results from the exploratory assessment will be discussed in a separate publication.

\section{Statistical Analysis}

Baseline was defined as the pre-dose measurement collected $45 \mathrm{~min}$ prior to dosing in each treatment period. The efficacy population consisted of patients who received $\geq 1$ dose of study drug and had a baseline and $\geq 1$ postbaseline RV measurement within the same period. The safety population consisted of all patients who received $\geq 1$ dose of study drug. Patients were analyzed according to randomized treatment group. All $p$ values and 95\% confidence intervals were two-sided; no multiplicity adjustments were made for treatment comparisons. All statistical procedures were performed using SAS ${ }^{\circledR}$ version 9.4 (SAS Institute Inc., Cary, NC, USA).

The sample size for this study was determined by a two-sample $t$ test (cross-over ANOVA) using nQuery version 4.0 (nQuery + nTerim version 4.0, Statistical Solutions, Cork,
Ireland). A sample size of ten evaluable patients per treatment sequence (20 patients in total), was required to achieve $88 \%$ power to detect a mean difference in the change from baseline in RV of - 0.31 (single dose of GLY compared with placebo), assuming a standard deviation (SD) of the differences of $0.4 \mathrm{l}$, with a one-sided significance level of 0.025 .

For the analysis of the primary endpoint, data were analyzed using a two-way crossover analysis of covariance (ANCOVA) using the efficacy population. The ANCOVA model included terms for treatment, period and sequence as fixed effects, period baseline as a covariate, and patients nested within sequence as a random effect. The Kenward and Roger correction for the degrees of freedom was used. Least squares (LS) mean difference in the change from baseline in $\mathrm{RV}$ at 6 -h post-treatment was calculated from the primary ANCOVA model. LS mean [and 95\% confidence interval (CI)] for each treatment group, and LS mean difference (and 95\% CI), and the associated $p$ value for the difference between the single dose of GLY and placebo were estimated.

Changes from baseline in RV, ERV, IC, FRC, TLC, sRaw, Raw, $\mathrm{FEV}_{1}$, and FVC up to 6-h posttreatment were analyzed similarly. Potential outliers among the 22 patients were evaluated using Tukey's 3 interquartile range (IQR) method after visual inspection of the unblinded data [27]. The primary analysis was then repeated post hoc after excluding any outliers.

\section{RESULTS}

\section{Patient Demographics and Baseline Characteristics}

A total of 44 patients were screened, of which 22 $(50.0 \%)$ were enrolled in the study (Fig. 2). Patient demographics and baseline characteristics are summarized in Table 1. Patients were all white, $54.5 \%$ female, with a mean age of 65.9 years. At baseline, 18/22 (81.8\%) patients had an mMRC score $\geq 3$; mean pre- and postbronchodilator RV \%predicted were 153.7 and $123.8 \%$, respectively. Two patients terminated the study early, after completing the GLY 


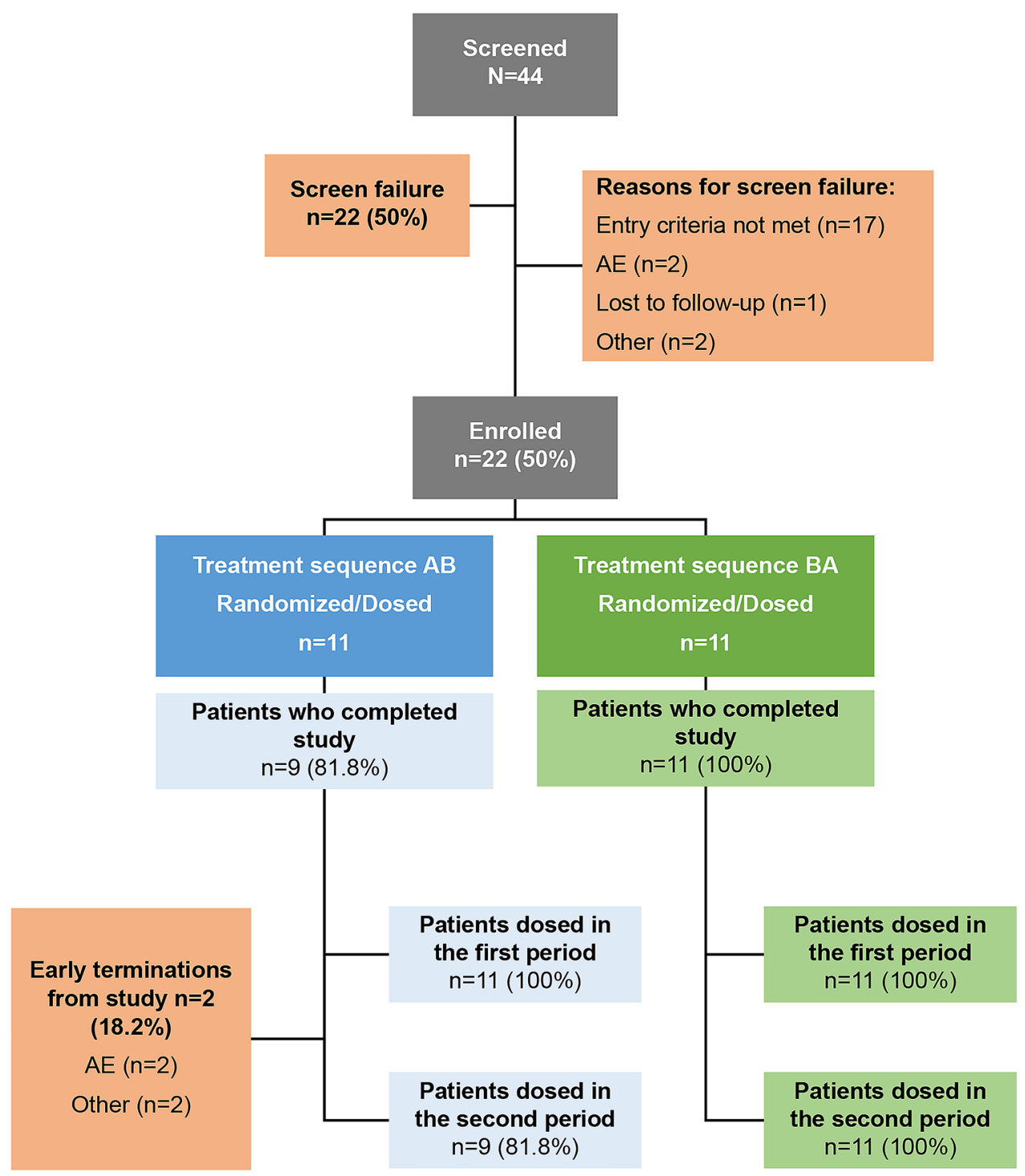

Fig. 2 Patient disposition in the SUN101-402 phase IV study. Treatment sequence $A B$ involved administration of GLY $25 \mu \mathrm{g}$ in treatment period 1 , and placebo in treatment period 2. Treatment sequence BA involved administration of placebo in treatment period 1, and GLY

treatment period, but before the placebo treatment period, one due to $\mathrm{AE}$ and one due to other reasons (COVID-19). As such, the placebo period data is missing for these two subjects.

\section{Efficacy}

Nebulized GLY was associated with improvements from baseline in RV at all time-points
$25 \mu \mathrm{g}$ in treatment period 2. $A E$ adverse event, $G L Y$ glycopyrrolate

analyzed in this study (Fig. 3a). Improvements in RV were observed with GLY relative to placebo at each hourly time-point post-treatment; however, statistical significance was not reached at the primary endpoint (6-h posttreatment). The placebo-adjusted LS mean (95\% $\mathrm{CI}$ ) change from baseline in $\mathrm{RV}$ at 6 -h posttreatment was $-0.3231(-0.711$ to 0.066 ; $p=0.0987$; Fig. 3a). These results demonstrate 
Table 1 Baseline demographics and clinical characteristics

\begin{tabular}{|c|c|}
\hline Parameter, mean (SD) & $\begin{array}{l}\text { Patients } \\
(N=22)\end{array}$ \\
\hline Age, years & $65.9(7.9)$ \\
\hline Female, $n(\%)$ & $12(54.5)$ \\
\hline BMI, $\mathrm{kg} / \mathrm{m}^{2}$ & $32.4(6.9)$ \\
\hline Race, white, $n(\%)$ & $22(100.0)$ \\
\hline Current smoker, $n(\%)$ & $11(50.0)$ \\
\hline Number of pack-years & $52.9(24.0)$ \\
\hline Pre-BD FEV 1 \%predicted & $51.9(12.2)$ \\
\hline Post-BD FEV $1 \%$ predicted & $59.7(13.2)$ \\
\hline Pre-BD FEV 1 /FVC, \% & $51.1(8.9)$ \\
\hline Post-BD FEV 1 /FVC, \% & $52.7(9.2)$ \\
\hline Pre-BD RV \%predicted & $153.7(20.4)$ \\
\hline Post-BD RV \%predicted & $123.8(23.9)$ \\
\hline $\begin{array}{l}\text { COPD exacerbations within } 12 \text { months } \\
\text { prior to screening }\end{array}$ & $0.2(0.5)$ \\
\hline \multicolumn{2}{|l|}{ mMRC, $n(\%)$} \\
\hline 2 & $4(18.2)$ \\
\hline 3 & $15(68.2)$ \\
\hline 4 & $3(13.6)$ \\
\hline SGRQ total scores & $43.8(13.9)$ \\
\hline Symptoms & $58.9(22.2)$ \\
\hline Activity & $59.6(18.8)$ \\
\hline Impacts & $30.1(14.0)$ \\
\hline
\end{tabular}

$B D$ bronchodilator, $B M I$ body mass index, $C O P D$ chronic obstructive pulmonary disease, $F E V_{1}$ forced expiratory volume in one second, $F V C$ forced vital capacity, $m M R C$ modified Medical Research Council dyspnea scale, $R V$ residual volume, SGRQ St George's Respiratory Questionnaire

clinically significant improvements in RV with nebulized GLY up to $6 \mathrm{~h}$. The trend over time demonstrated meaningful improvements.

An evaluation of change from baseline in RV at 6-h post-treatment in individual patients (Fig. 3c, d) identified a statistical outlier value from one patient (65-year-old female) treated with GLY during treatment period 2 . The outlier value of $2.41 \mathrm{l}$ was identified based on Tukey's 3 IQR method. When the primary analysis was repeated post hoc after excluding this outlier, placebo-adjusted LS mean (95\% CI) change from baseline in RV was $-0.446 \mathrm{l}(-0.741$ to - 0.150; Fig. 3b).

\section{Other Efficacy Endpoints}

At 6-h post-treatment, improvements with GLY compared with placebo were observed in both spirometric $\left(\mathrm{FEV}_{1}\right.$ and FVC; Fig. 4a) and plethysmographic (IC, sRaw, and Raw) parameters (Fig. 4b-d). Change from baseline in plethysmography endpoints were also assessed at $1,2,3$, and $4 \mathrm{~h}$ post-treatment; RV and IC were the only endpoints to achieve notable improvements at all time-points tested (Tables S1, S2). For RV, IC, FRC, and sRaw, improvements from baseline with GLY compared with placebo were also observed in AUC from time zero to 3, 4 , and $6 \mathrm{~h}$ post-treatment (Tables 2,3 ).

Pearson's correlations ( $r$ ) between changes in spirometric measures ( $\mathrm{FEV}_{1}$ or FVC) with those in plethysmographic measures at $6-\mathrm{h}$ posttreatment were estimated. Moderate correlations $(95 \% \mathrm{CI})$ between changes from baseline in $\mathrm{FEV}_{1}$ in the GLY treatment arm were observed with several plethysmographic measures, including RV $[r=-0.535(-0.78$ to $-0.15)]$, and IC $[r=0.726(0.44-0.88)]$; similar correlations were also observed with FVC (Table S3).

\section{Safety}

GLY was generally well tolerated, with a low incidence of AEs and AEs leading to study discontinuation (Table 4). One AE of COPD worsening (moderate intensity and unrelated to the study medication) was reported in a patient treated with a single dose of GLY, which led to study discontinuation. There was $1 \mathrm{SAE}$ (COPD worsening) reported during the screening period, and the patient withdrew from the study before drug administration. There were no 
a

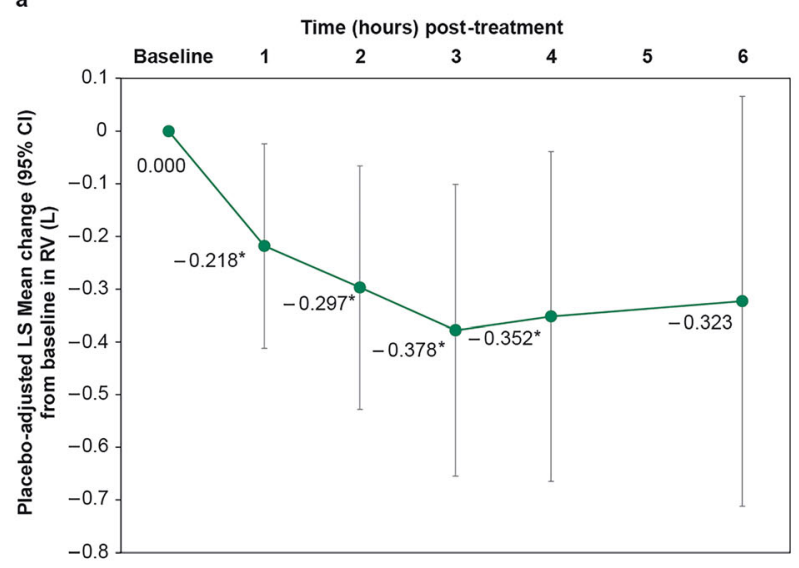

c

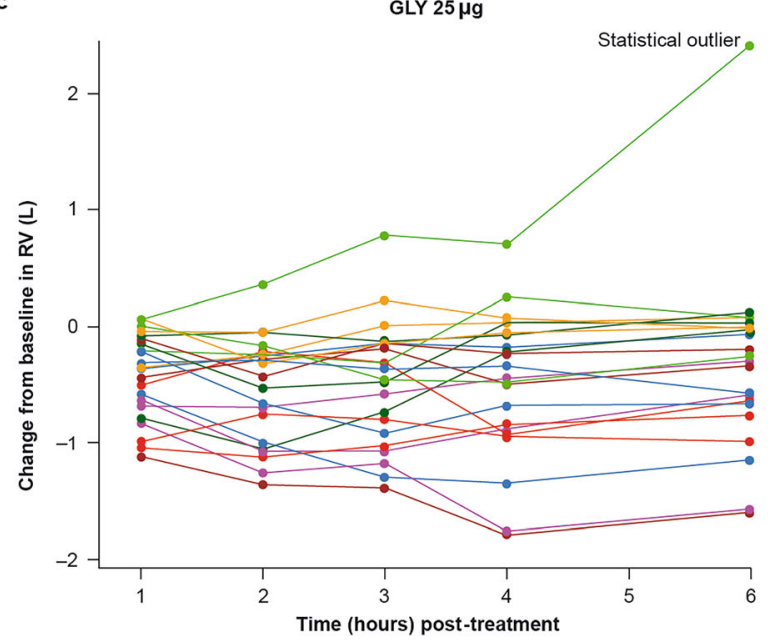

Fig. 3 Change from baseline in residual volume a with treatment time in the efficacy population, $\mathbf{b}$ at the primary endpoint in either the efficacy population or after exclusion of the outlier, and for individual patients treated with c GLY or d placebo. ${ }^{*} p<0.05 ;{ }^{* *} p<0.01$ vs.

deaths or SAEs reported during the randomized treatment period.

\section{DISCUSSION}

This is the first study to evaluate the effect of a single dose of nebulized LAMA (GLY $25 \mu \mathrm{g}$ ) on hyperinflation in patients with COPD. The primary endpoint of this study, RV improvement at $6 \mathrm{~h}$ post-GLY nebulization, was not met; however, at all time-points assessed in this b

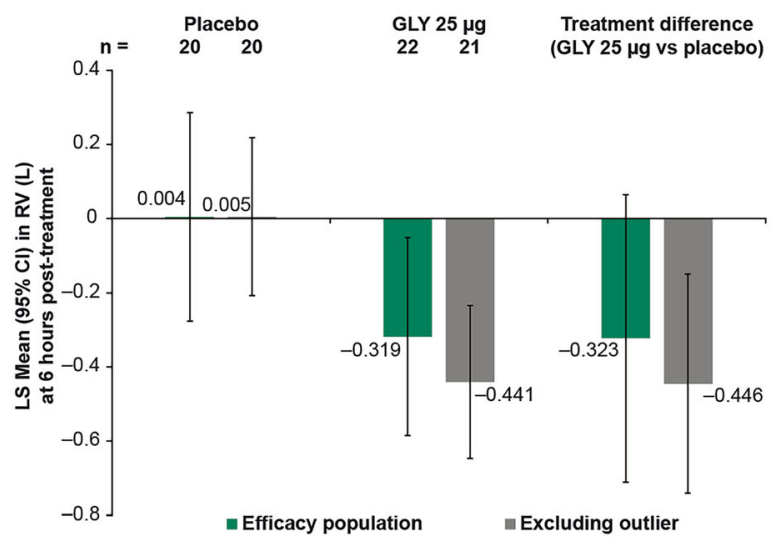

d

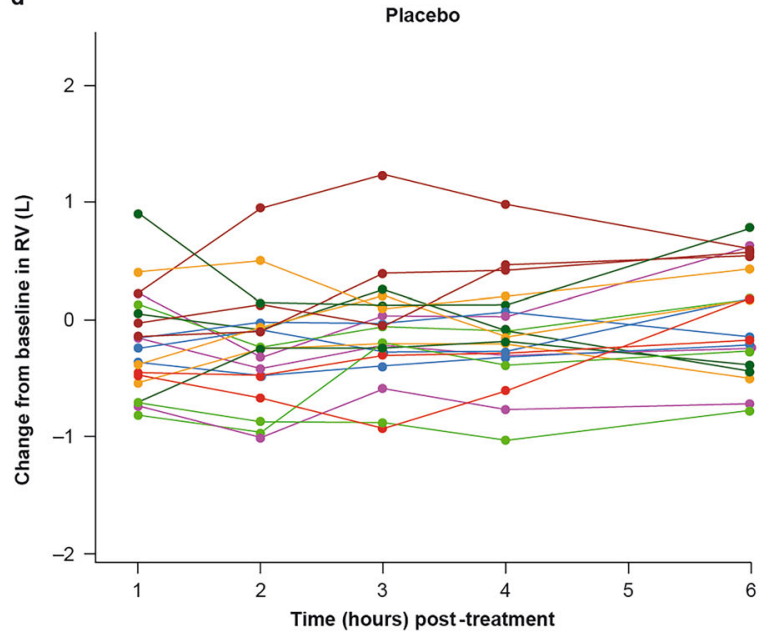

placebo. $C I$ confidence interval, GLY nebulized glycopyrrolate, $L S$ least squares, $R V$ residual volume

study, a single dose of GLY showed marked improvements in RV compared with placebo. The placebo-adjusted LS mean change from baseline in RV with GLY were $-0.218,-0.297$, -0.378 , and $-0.352 \mathrm{l}$, at $1,2,3$, and $4 \mathrm{~h}$ posttreatment, respectively $(p<0.05$ at all timepoints). At 6 -h post-treatment (primary endpoint), the placebo-adjusted LS mean change from baseline was - $0.323 \mathrm{l}$; however, it did not reach statistical significance at the 5\% level. After excluding a single statistical outlier value 

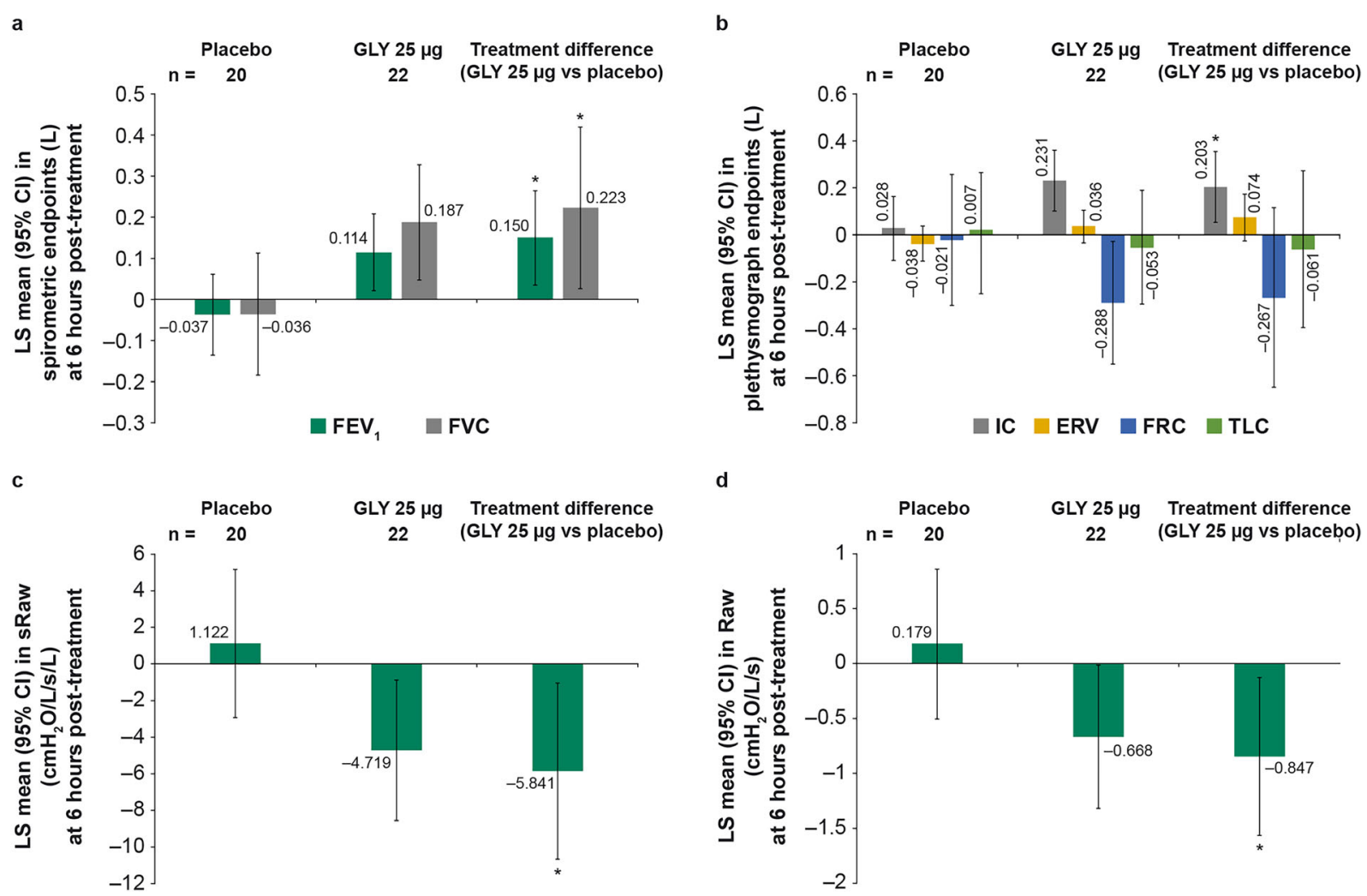

Fig. 4 Change from baseline in spirometric (a), plethysmographic (b) endpoints, specific airway resistance (c), and airways resistance (d) at 6 -h post-treatment, in the efficacy population. ${ }^{*} p<0.05$ vs. placebo. $C I$ confidence interval, $E R V$ expiratory reserve volume, $F E V_{1}$ forced expiratory volume in $1 \mathrm{~s}, F R C$ functional residual capacity, $F V C$

forced vital capacity, $G L Y$ glycopyrrolate, $I C$ inspiratory capacity, $l$ liter(s), LS least squares, Raw airway resistance, $R V$ residual volume, sRaw specific airway resistance, TLC total lung capacity

(change from baseline in RV of $2.41 \mathrm{l}$ ) based on Tukey's 3 IQR method, a post hoc analysis of the primary endpoint showed greater improvements in RV (placebo-adjusted LS mean change from baseline of $-0.446 \mathrm{l}$ ). AUC analysis for RV performed at $0-3,0-4$, and $0-6 \mathrm{~h}$ post-treatment showed improvements in placebo-adjusted change from baseline with GLY $25 \mu \mathrm{g}$, which suggests a rapid reduction in hyperinflation after the first dose. Further, standardized changes from baseline in $\mathrm{AUC}_{0-3 \mathrm{~h}}, \mathrm{AUC}_{0-4 \mathrm{~h}}$, and $\mathrm{AUC}_{0-6} \mathrm{~h}$ for IC, FRC, and sRaw demonstrated improvements at all three time-points $(p<0.05)$. These results suggest that AUC measures may be more valuable than single time-points, when assessing improvements after a single dose.

It is important to note that the reduction in RV was also accompanied by an increase in IC, and a reduction in airway resistance (Raw and sRaw), whereas the TLC remained generally unchanged following treatment. Spirometric measures (FEV 1 and FVC) also showed improvements from baseline with GLY compared with placebo at 6-h post-treatment; these improvements correlated negatively (with changes in RV, FRC, Raw, sRaw), and positively (with changes in IC). Together, these results demonstrate that a single dose of nebulized GLY $25 \mu \mathrm{g}$ may improve functional lung capacity as early as 6-h post-treatment in patients with COPD and hyperinflation.

Previous studies with LAMAs delivered via DPIs have demonstrated lung deflation in 
Table 2 LS mean (SE) standardized change from baseline in AUC of plethysmography endpoints by treatment (efficacy population)

\begin{tabular}{|c|c|c|c|c|c|c|}
\hline \multirow[t]{2}{*}{ Parameter $(\mathbf{L})$} & \multirow[t]{2}{*}{ Time-points } & \multirow{2}{*}{$\begin{array}{l}\text { Placebo } \\
N=20 \\
\text { LS mean (SE) }\end{array}$} & \multirow{2}{*}{$\begin{array}{l}\text { GLY } 25 \mu \mathrm{g} \\
N=22 \\
\text { LS mean (SE) }\end{array}$} & \multicolumn{3}{|c|}{ Treatment difference from placebo } \\
\hline & & & & LS mean (SE) & $95 \% \mathrm{CI}$ & $p$ value \\
\hline \multirow[t]{3}{*}{ RV } & $\mathrm{AUC}_{0-3 \mathrm{~h}}$ & $-0.1625(0.0709)$ & $-0.3987(0.0675)$ & $-0.2362(0.0765)$ & $-0.3984,-0.0740$ & 0.0071 \\
\hline & $\mathrm{AUC}_{0-4 \mathrm{~h}}$ & $-0.1508(0.0779)$ & $-0.4189(0.0739)$ & $-0.2681(0.0893)$ & $-0.4566,-0.0796$ & 0.0081 \\
\hline & $\mathrm{AUC}_{0-6 \mathrm{~h}}$ & $-0.1220(0.0892)$ & $-0.4132(0.0843)$ & $-0.2911(0.1097)$ & $-0.5215,-0.0607$ & 0.0161 \\
\hline \multirow[t]{3}{*}{ IC } & $\mathrm{AUC}_{0-3 \mathrm{~h}}$ & $-0.0031(0.0453)$ & $0.2123(0.0431)$ & $0.2155(0.0521)$ & $0.1064,0.3245$ & 0.0006 \\
\hline & $\mathrm{AUC}_{0-4 \mathrm{~h}}$ & $-0.0037(0.0517)$ & $0.2250(0.0493)$ & $0.2288(0.0563)$ & $0.1106,0.3470$ & 0.0007 \\
\hline & $\mathrm{AUC}_{0-6 \mathrm{~h}}$ & $0.0013(0.0560)$ & $0.2292(0.0536)$ & $0.2279(0.0585)$ & $0.1050,0.3508$ & 0.0011 \\
\hline \multirow[t]{3}{*}{ ERV } & $\mathrm{AUC}_{0-3 \mathrm{~h}}$ & $-0.0111(0.0238)$ & $0.0337(0.0226)$ & $0.0448(0.0330)$ & $-0.0218,0.1113$ & 0.1812 \\
\hline & $\mathrm{AUC}_{0-4 \mathrm{~h}}$ & $-0.0224(0.0246)$ & $0.0267(0.0234)$ & $0.0491(0.0339)$ & $-0.0197,0.1179$ & 0.1565 \\
\hline & $\mathrm{AUC}_{0-6 \mathrm{~h}}$ & $-0.0303(0.0262)$ & $0.0269(0.0247)$ & $0.0572(0.0358)$ & $-0.0179,0.1322$ & 0.1272 \\
\hline \multirow[t]{3}{*}{ FRC } & $\mathrm{AUC}_{0-3 \mathrm{~h}}$ & $-0.1461(0.0692)$ & $-0.3920(0.0656)$ & $-0.2459(0.0781)$ & $-0.4106,-0.0811$ & 0.0059 \\
\hline & $\mathrm{AUC}_{0-4 \mathrm{~h}}$ & $-0.1446(0.0758)$ & $-0.4174(0.0717)$ & $-0.2728(0.0892)$ & $-0.4605,-0.0852$ & 0.0069 \\
\hline & $\mathrm{AUC}_{0-6 \mathrm{~h}}$ & $-0.1241(0.0863)$ & $-0.4071(0.0815)$ & $-0.2830(0.1084)$ & $-0.5101,-0.0559$ & 0.0173 \\
\hline \multirow[t]{3}{*}{ TLC } & $\mathrm{AUC}_{0-3 \mathrm{~h}}$ & $-0.1466(0.0566)$ & $-0.1796(0.0537)$ & $-0.0330(0.0667)$ & $-0.1727,0.1068$ & 0.6265 \\
\hline & $\mathrm{AUC}_{0-4 \mathrm{~h}}$ & $-0.1449(0.0619)$ & $-0.1920(0.0586)$ & $-0.0472(0.0747)$ & $-0.2038,0.1095$ & 0.5357 \\
\hline & $\mathrm{AUC}_{0-6 \mathrm{~h}}$ & $-0.1200(0.0708)$ & $-0.1766(0.0670)$ & $-0.0565(0.0884)$ & $-0.2414,0.1284$ & 0.5303 \\
\hline
\end{tabular}

$A U C$ area under the curve, $C I$ confidence interval, $E R V$ expiratory reserve volume, $F R C$ functional residual capacity, $G L Y$ glycopyrrolate, $I C$ inspiratory capacity, $l$ liter(s), $L S$ least squares, $R V$ residual volume, $S E$ standard error, $T L C$ total lung capacity

COPD. In a study of 20 patients with stable COPD and hyperinflation, DPI-mediated administration of tiotropium (a LAMA) or budesonide/formoterol (inhaled corticosteroid [ICS]/LABA) showed improvements in spirometry and hyperinflation in both treatment groups [28]. In a study of 37 patients with severe-tovery-severe COPD with hyperinflation (RV $>125 \%$ of predicted normal), two LAMAsaclidinium bromide $400 \mu \mathrm{g}$ or glycopyrronium $50 \mu \mathrm{g}$ QD (both delivered via DPIs) - reduced hyperinflation and dyspnea; reduction in RV with aclidinium bromide $400 \mu \mathrm{g}$ occurred earlier (5-180 min post-treatment), compared with glycopyrronium $50 \mu \mathrm{g}$ (60 and $180 \mathrm{~min}$ ) [14]. The improvements in lung hyperinflation observed in the current study are comparable to those reported with single doses of LAMA delivered via DPIs [14].
Recent studies with long-acting bronchodilators have demonstrated the positive impact of lung deflation on cardiac function in COPD patients with static hyperinflation [15, 29-32]. Treatment with indacaterol (a LABA; $150 \mu \mathrm{g}$ ) in 40 patients with stable COPD and $\mathrm{RV} \geq 135 \%$ of predicted normal, showed reductions in hyperinflation in acute conditions, compared with placebo; further, this was associated with improvements in cardiac function [15]. The CLAIM study analyzed the effect of dual bronchodilation (LABA + LAMA combination: indacaterol $110 \mu \mathrm{g} / \mathrm{glycopyrronium}$ $50 \mu \mathrm{g})$ vs. placebo on cardiac function, in 62 patients with COPD and hyperinflation [29]. Following 14 days of treatment, indacaterol/ glycopyrronium significantly improved cardiac function (left-ventricular end-diastolic volume) and $\mathrm{FEV}_{1}$, and reduced hyperinflation, when compared with placebo [29]. While cardiac 
Table 3 LS mean (SE) standardized change from baseline in AUC of plethysmography airway resistance by treatment (efficacy population)

\begin{tabular}{|c|c|c|c|c|c|c|}
\hline \multirow[t]{2}{*}{ Parameter } & \multirow[t]{2}{*}{ Time-points } & \multirow{2}{*}{$\begin{array}{l}\text { Placebo } \\
N=20 \\
L S \text { mean }(\mathrm{SE})\end{array}$} & \multirow{2}{*}{$\begin{array}{l}\text { GLY } 25 \mu \mathrm{g} \\
N=22 \\
\text { LS mean }(\mathrm{SE})\end{array}$} & \multicolumn{3}{|c|}{ Treatment difference from placebo } \\
\hline & & & & LS mean (SE) & 95\% CI & $p$ value \\
\hline \multirow[t]{3}{*}{ sRaw } & $\mathrm{AUC}_{0-3 \mathrm{~h}}$ & $0.0537(1.3522)$ & $-4.9773(1.2759)$ & & $-8.8019,-1.2601$ & 0.0117 \\
\hline & $\mathrm{AUC}_{0-4 \mathrm{~h}}$ & $0.1384(1.4499)$ & $-5.0493(1.3690)$ & $-5.1877(1.8757)$ & $-9.1250,-1.2503$ & 0.0126 \\
\hline & $\mathrm{AUC}_{0-6 \mathrm{~h}}$ & $0.3096(1.5985)$ & $-4.9259(1.5115)$ & $-5.2354(1.9951)$ & $-9.4246,-1.0463$ & 0.0171 \\
\hline \multirow[t]{3}{*}{ Raw } & $\mathrm{AUC}_{0-3 \mathrm{~h}}$ & $0.1261(0.2636)$ & $-0.6077(0.2501)$ & $-0.7338(0.3638)$ & $-1.4708,0.0033$ & 0.0510 \\
\hline & $\mathrm{AUC}_{0-4 \mathrm{~h}}$ & $0.1326(0.2727)$ & $-0.6033(0.2571)$ & $-0.7360(0.3700)$ & $-1.5109,0.0390$ & 0.0614 \\
\hline & $\mathrm{AUC}_{0-6 \mathrm{~h}}$ & $0.1369(0.2879)$ & $-0.5955(0.2719)$ & $-0.7324(0.3671)$ & $-1.5016,0.0368$ & 0.0608 \\
\hline
\end{tabular}

$A U C$ area under the curve, $C I$ confidence interval, $G L Y$ glycopyrrolate, $L S$ least squares, Raw airway resistance $\left(\mathrm{cmH}_{2} \mathrm{O} / \mathrm{l} /\right.$ s), $S E$ standard error, sRaw specific airway resistance $\left(\mathrm{cmH}_{2} \mathrm{O} / \mathrm{l} / \mathrm{s} / \mathrm{l}\right)$

Table 4 Summary of AEs, SAEs, and AEs leading to study discontinuation by treatment group (safety population)

\begin{tabular}{lll}
\hline & $\begin{array}{l}\text { Placebo } \\
\boldsymbol{N}=\mathbf{2 0}\end{array}$ & $\begin{array}{l}\text { GLY } \\
\mathbf{2 5} \boldsymbol{\mu g} \\
\boldsymbol{N}=\mathbf{2 2}\end{array}$ \\
\hline Any AE & 0 & $1(4.5)$ \\
Worsening of COPD & 0 & $1(4.5)$ \\
Any SAE & 0 & 0 \\
$\begin{array}{l}\text { AE leading to study } \\
\text { discontinuation }\end{array}$ & 0 & $1(4.5)$ \\
\hline
\end{tabular}

$A E$ adverse event, $C O P D$ chronic obstructive pulmonary disease, $S A E$ serious adverse event

functions were not assessed in this study, it is possible that the improvements in RV and other lung function parameters noted with nebulized GLY may be associated with improvements in cardiac function, similar to other trials.

A retrospective analysis conducted in 2265 patients with COPD showed that in patients with mild COPD (GOLD stage I), RV was $\sim 135 \%$ of predicted normal, and sRaw was $\sim 250 \%$ of predicted normal [3]. This is an important observation because it demonstrates that significant air trapping and hyperinflation can occur even with mild COPD. Patients with mild COPD are typically excluded from clinical studies of COPD; however, for characterization of the impact of bronchodilators on hyperinflation, inclusion of GOLD I patients may be highly informative. This study also highlights the importance of serial lung volume measurements with body plethysmography in the diagnosis/treatment of hyperinflation in COPD. While plethysmography is not typically done in clinical practice, it can provide valuable insight for treating hyperinflation in patients with COPD. Many patients with COPD experience nocturnal symptoms [33]; though not the objective of this study, it would be interesting to evaluate if an additional evening dose of GLY has any benefits on static lung hyperinflation and nocturnal symptoms.

This study had a few limitations. The primary endpoint of this study (6-h post-treatment) may have been too early to detect maximal impacts on lung deflation. Further, a single-dose of GLY $25 \mu \mathrm{g}$ was used in this study, and not the FDA-approved dose ( $25 \mu \mathrm{g}$ BID). This study was conducted to understand the impact of a single dose of GLY $25 \mu \mathrm{g}$ on lung hyperinflation. Assessments beyond $6 \mathrm{~h}$ of treatment, combined with the complete dose of GLY for longer periods of time, may provide insights into the long-term impact of nebulized GLY on lung hyperinflation in COPD. In addition, the relatively small sample size in this study may have limited the detection of safety signals and led to the undue influence of the 
single statistical outlier value in the primary analysis. Regarding the statistical outlier, this patient did not have any known measurement or data entry errors nor did the patient have any characteristics that would result in their exclusion from the analysis. As such, we did not exclude this data point from the primary analysis; but we did perform an additional post hoc analysis excluding this data point to show its impact on the results. Despite not reaching statistical significance in the primary analysis $(p=0.0987)$, the placebo-adjusted improvement in RV of $-0.323 \mathrm{l}$ did exceed the $0.3 \mathrm{l}$ minimal clinically important difference [34], underscoring that a clinically meaningful outcome for this patient population was observed.

\section{CONCLUSIONS}

This was the first randomized study to evaluate the impact of a single dose of a nebulized LAMA (GLY) on lung hyperinflation. A single dose of nebulized GLY $25 \mu \mathrm{g}$ showed clinically important improvements in measures of lung hyperinflation up to 6-h post-treatment, compared with placebo. Further, notable improvements in both spirometric $\left(\mathrm{FEV}_{1}\right)$ and plethysmographic measures were observed up to 6 -h post-treatment. This study highlights the potential of nebulized LAMAs in reducing lung hyperinflation in patients with COPD.

\section{ACKNOWLEDGEMENTS}

The authors would like to thank all patients who participated in this study.

Funding. This study and the journal's Rapid Service Fee were funded by Sunovion Pharmaceuticals Inc.

Medical writing assistance. Medical writing support for the development of this manuscript, under the direction of the authors, was provided by Dhivya Ramalingam, PhD and Hashem Dbouk, PhD of Ashfield MedComms, an Ashfield Health company, and funded by Sunovion Pharmaceuticals Inc.
Authorship. All named authors meet the International Committee of Medical Journal Editors (ICMJE) criteria for authorship for this article, take responsibility for the integrity of the work as a whole, and have given their approval for this version to be published.

Authors' contributions. TMS, CH, SSa, and SSh contributed to protocol development and design. TMS was involved in data acquisition. TMS, CH, KX, KS, SSa and SSh were involved in data analysis and interpretation. TMS, $\mathrm{CH}, \mathrm{KX}$, $\mathrm{KS}$, SSa and SSh were involved at all stages of manuscript development, writing, and revision.

Disclosures. Thomas M Siler received research support from West-Ward Pharmaceuticals, Theravance Biopharma US, Inc., GlaxoSmithKline plc., Pearl Therapeutics, Chiesi, AstraZeneca, Novartis, Boehringer Ingelheim, Forest, Compleware, Evidera, Oncocyte, Teva, Vapotherm, Sunovion, Proterix BioPharma, Seer, Sanofi and Verona Pharma. Thomas M Siler has also received speaker fees from GlaxoSmithKline plc., Mylan Inc./Theravance Biopharma US, Inc., and Sunovion, and consulting fees from Vapotherm. Claire Hohenwarter, Kuangnan Xiong, Kenneth Sciarappa, Shahin Sanjar and Sanjay Sharma are employees of Sunovion Pharmaceuticals Inc.

Compliance with ethics guidelines. The study (SUN101-402: project approval number 39173) protocol was approved by Quorum Review institutional review board prior to enrollment and was conducted in accordance with the protocols, International Conference on Harmonization Good Clinical Practice guidelines, and Declaration of Helsinki. All patients provided written informed consent before participating in this study.

Data availability. Sunovion Pharmaceuticals Inc. is part of a clinical trial data sharing consortium that facilitates access for qualified researchers to selected anonymized clinical trial data. For up-to-date information on data availability, please visit: https://www. clinicalstudydatarequest.com/Study-Sponsors. aspx and click on Sunovion. 
Open Access. This article is licensed under a Creative Commons Attribution-NonCommercial 4.0 International License, which permits any non-commercial use, sharing, adaptation, distribution and reproduction in any medium or format, as long as you give appropriate credit to the original author(s) and the source, provide a link to the Creative Commons licence, and indicate if changes were made. The images or other third party material in this article are included in the article's Creative Commons licence, unless indicated otherwise in a credit line to the material. If material is not included in the article's Creative Commons licence and your intended use is not permitted by statutory regulation or exceeds the permitted use, you will need to obtain permission directly from the copyright holder. To view a copy of this licence, visit http://creativecommons.org/licenses/by$\mathrm{nc} / 4.0 /$.

\section{REFERENCES}

1. Global Initiative for Chronic Obstructive Lung Disease (GOLD). Global Strategy for the Diagnosis, Management, and Prevention of Chronic Obstructive Pulmonary Disease. 2021. Available from: https://goldcopd.org/2021-gold-reports/. Accessed 23 Mar 2021.

2. Vestbo J, Hurd SS, Agusti AG, Jones PW, Vogelmeier C, Anzueto A, et al. Global strategy for the diagnosis, management, and prevention of chronic obstructive pulmonary disease: GOLD executive summary. Am J Respir Crit Care Med. 2013;187(4): 347-65.

3. Deesomchok A, Webb KA, Forkert L, Lam YM, Ofir $\mathrm{D}$, Jensen $\mathrm{D}$, et al. Lung hyperinflation and its reversibility in patients with airway obstruction of varying severity. COPD. 2010;7(6):428-37.

4. Rossi A, Aisanov Z, Avdeev S, Di Maria G, Donner $\mathrm{CF}$, Izquierdo JL, et al. Mechanisms, assessment and therapeutic implications of lung hyperinflation in COPD. Respir Med. 2015;109(7):785-802.

5. Watz H, Waschki B, Meyer T, Kretschmar G, Kirsten A, Claussen $M$, et al. Decreasing cardiac chamber sizes and associated heart dysfunction in COPD: role of hyperinflation. Chest. 2010;138(1):32-8.

6. Smith BM, Kawut SM, Bluemke DA, Basner RC, Gomes AS, Hoffman E, et al. Pulmonary hyperinflation and left ventricular mass: the MultiEthnic Study of Atherosclerosis COPD Study. Circulation. 2013;127(14):1503-11 (11e1-6).

7. Langer D, Ciavaglia CE, Neder JA, Webb KA, O'Donnell DE. Lung hyperinflation in chronic obstructive pulmonary disease: mechanisms, clinical implications and treatment. Expert Rev Respir Med. 2014;8(6):731-49.

8. Beeh KM, Watz H, Puente-Maestu L, de Teresa L, Jarreta D, Caracta C, et al. Aclidinium improves exercise endurance, dyspnea, lung hyperinflation, and physical activity in patients with COPD: a randomized, placebo-controlled, crossover trial. BMC Pulm Med. 2014;14:209.

9. Watz H, Troosters T, Beeh KM, Garcia-Aymerich J, Paggiaro P, Molins E, et al. ACTIVATE: the effect of aclidinium/formoterol on hyperinflation, exercise capacity, and physical activity in patients with COPD. Int J Chron Obstr Pulm Dis. 2017;12: 2545-58.

10. Casaburi R, Maltais F, Porszasz J, Albers F, Deng Q, Iqbal A, et al. Effects of tiotropium on hyperinflation and treadmill exercise tolerance in mild to moderate chronic obstructive pulmonary disease. Ann Am Thorac Soc. 2014;11(9):1351-61.

11. Gelb AF, Taylor CF, Cassino C, Shinar CM, Schein MJ, Zamel N. Tiotropium induced bronchodilation and protection from dynamic hyperinflation is independent of extent of emphysema in COPD. Pulm Pharmacol Ther. 2009;22(3):237-42.

12. O'Donnell DE, Fluge $\mathrm{T}$, Gerken $\mathrm{F}$, Hamilton $\mathrm{A}$, Webb K, Aguilaniu B, et al. Effects of tiotropium on lung hyperinflation, dyspnoea and exercise tolerance in COPD. Eur Respir J. 2004;23(6):832-40.

13. Verkindre C, Bart F, Aguilaniu B, Fortin F, Guerin JC, Le Merre C, et al. The effect of tiotropium on hyperinflation and exercise capacity in chronic obstructive pulmonary disease. Respiration. 2006;73(4):420-7.

14. Santus P, Radovanovic D, Di Marco F, Raccanelli R, Valenti V, Centanni S. Faster reduction in hyperinflation and improvement in lung ventilation inhomogeneity promoted by aclidinium compared to glycopyrronium in severe stable COPD patients. A randomized crossover study. Pulm Pharmacol Ther. 2015;35:42-9.

15. Santus P, Radovanovic D, Di Marco S, Valenti V, Raccanelli R, Blasi F, et al. Effect of indacaterol on lung deflation improves cardiac performance in hyperinflated COPD patients: an interventional, randomized, double-blind clinical trial. Int J Chron Obstr Pulm Dis. 2015;10:1917-23. 
16. Maltais F, Celli B, Casaburi R, Porszasz J, Jarreta D, Seoane B, et al. Aclidinium bromide improves exercise endurance and lung hyperinflation in patients with moderate to severe COPD. Respir Med. 2011;105(4):580-7.

17. Santus P, Radovanovic D, Cristiano A, Valenti V, Rizzi M. Role of nebulized glycopyrrolate in the treatment of chronic obstructive pulmonary disease. Drug Des Dev Ther. 2017;11:3257-71.

18. Pham S, Ferguson GT, Kerwin E, Goodin T, Wheeler A, Bauer A. In vitro characterization of the eFlow closed system nebulizer with glycopyrrolate inhalation solution. J Aerosol Med Pulm Drug Deliv. 2018;31(3):162-9.

19. Sunovion Pharmaceuticals Inc. Lonhala Magnair (glycopyrrolate) inhalation solution: highlights of prescribing information (2019).

20. Kerwin E, Donohue JF, Goodin T, Tosiello R, Wheeler A, Ferguson GT. Efficacy and safety of glycopyrrolate/eFlow((R)) CS (nebulized glycopyrrolate) in moderate-to-very-severe COPD: Results from the glycopyrrolate for obstructive lung disease via electronic nebulizer (GOLDEN) 3 and 4 randomized controlled trials. Respir Med. 2017;132: 238-50.

21. Ferguson GT, Goodin T, Tosiello R, Wheeler A, Kerwin E. Long-term safety of glycopyrrolate/ eFlow $((\mathrm{R})) \mathrm{CS}$ in moderate-to-very-severe COPD: results from the glycopyrrolate for obstructive lung disease via electronic nebulizer (GOLDEN) 5 randomized study. Respir Med. 2017;132:251-60.

22. Ohar JA, Bauer A, Sharma S, Sanjar S. In vitro effect of different airflow rates on the aerosol properties of nebulized glycopyrrolate in the eFlow(R) closed system and tiotropium delivered in the HandiHaler(R). Pulm Ther. 2020;6(2):289-301.

23. Laube BL, Janssens HM, de Jongh FH, Devadason SG, Dhand R, Diot P, et al. What the pulmonary specialist should know about the new inhalation therapies. Eur Respir J. 2011;37(6):1308-31.

24. Pleasants RA 2nd. Glycopyrrolate/eFlow CS: the first nebulized long-acting muscarinic antagonist approved to treat chronic obstructive pulmonary disease. Ann Pharmacother. 2019;53(3):285-93.

25. Miller MR, Hankinson J, Brusasco V, Burgos F, Casaburi R, Coates A, et al. Standardisation of spirometry. Eur Respir J. 2005;26(2):319-38.

26. Wanger J, Clausen JL, Coates A, Pedersen OF, Brusasco V, Burgos F, et al. Standardisation of the measurement of lung volumes. Eur Respir J. 2005;26(3):511-22.

27. Seo, S. A Review and Comparison of Methods for Detecting Outliers in Univariate Data Sets. Master's Thesis, University of Pittsburgh 2006. (Unpublished).

28. Santus P, Centanni S, Verga M, Di Marco F, Matera MG, Cazzola M. Comparison of the acute effect of tiotropium versus a combination therapy with single inhaler budesonide/formoterol on the degree of resting pulmonary hyperinflation. Respir Med. 2006;100(7):1277-81.

29. Hohlfeld JM, Vogel-Claussen J, Biller H, Berliner D, Berschneider K, Tillmann HC, et al. Effect of lung deflation with indacaterol plus glycopyrronium on ventricular filling in patients with hyperinflation and COPD (CLAIM): a double-blind, randomised, crossover, placebo-controlled, single-centre trial. Lancet Respir Med. 2018;6(5):368-78.

30. Herth F, Hohlfeld JM, Haas J, de la Hoz A, Jin X, Kreitner KF, et al. The effect of tiotropium/olodaterol versus fluticasone propionate/salmeterol on left ventricular filling and lung hyperinflation in patients with COPD. BMJ Open Respir Res. 2020;7(1):e000741.

31. Stone IS, Barnes NC, James WY, Midwinter D, Boubertakh R, Follows R, et al. Lung deflation and cardiovascular structure and function in chronic obstructive pulmonary disease. A randomized controlled trial. Am J Respir Crit Care Med. 2016;193(7):717-26.

32. Vogel-Claussen J, Schonfeld CO, Kaireit TF, Voskrebenzev A, Czerner CP, Renne J, et al. Effect of indacaterol/glycopyrronium on pulmonary perfusion and ventilation in hyperinflated patients with chronic obstructive pulmonary disease (CLAIM). A double-blind, randomized, crossover trial. Am J Respir Crit Care Med. 2019;199(9):1086-96.

33. Stephenson JJ, Cai Q, Mocarski M, Tan H, Doshi JA, Sullivan SD. Impact and factors associated with nighttime and early morning symptoms among patients with chronic obstructive pulmonary disease. Int J Chron Obstr Pulm Dis. 2015;10:577-86.

34. Hartman JE, Ten Hacken NH, Klooster K, Boezen $\mathrm{HM}$, de Greef MH, Slebos DJ. The minimal important difference for residual volume in patients with severe emphysema. Eur Respir J. 2012;40(5): 1137-41. 